\title{
DNA-dependent protein kinase: a potent inhibitor of transcription by RNA polymerase I
}

\author{
Anne Kuhn,' Tanya M. Gottlieb, ${ }^{2}$ Stephen P. Jackson, ${ }^{2,3}$ and Ingrid Grummt ${ }^{1}$ \\ ${ }^{1}$ German Cancer Research Centre, D-69120 Heidelberg, Germany; ${ }^{2}$ Wellcome/CRC Institute and Department of Zoology, \\ Cambridge University, Cambridge CB2 LQR, UK
}

\begin{abstract}
DNA-dependent protein kinase (DNA-PK) comprises a catalytic subunit of $\sim 350 \mathrm{kD}$ (p350) and a DNA-binding component termed $\mathrm{Ku}$. Although DNA-PK can phosphorylate many transcription factors, no function for this enzyme in transcription has been reported thus far. Here, we show that DNA-PK strongly represses transcription by RNA polymerase I (Pol I). Transcriptional repression by DNA-PK requires ATP hydrolysis, and DNA-PK must be colocalized on the same DNA molecule as the Pol I transcription machinery. Consistent with DNA-PK requiring DNA ends for activity, transcriptional inhibition only occurs effectively on linearized templates. Mechanistic studies including single-round transcriptions, abortive initiation assays, and factor-independent transcription on a tailed template demonstrate that DNA-PK inhibits initiation (i.e., the formation of the first phosphodiester bonds) but does not affect transcription elongation. Repression of transcription involves phosphorylation of the transcription initiation complex, and rescue experiments reveal that the inactivated factor remains bound to the promoter and thus prevents initiation complex formation. We discuss the possible relevance of these findings in regard to the control of rRNA synthesis in vivo.
\end{abstract}

[Key Words: DNA-PK; Ku; RNA polymerase I; transcription; transcription factor]

Received July 28, 1994; revised version accepted December 1, 1994.

As with class II and III promoters, transcriptional initiation by RNA polymerase I ( $\mathrm{Pol} \mathrm{I}$ ) involves the stepwise assembly of basal factors on the promoter to form a preinitiation complex. Transcription complex formation is nucleated by binding of the promoter selectivity factor, termed TIF-IB in rodents and SL1 in humans. TIF$\mathrm{IB} / \mathrm{SL} 1$ is a multiprotein complex containing the TATAbinding protein (TBP) and three TBP-associated factors (polypeptides) (TAFs) (Comai et al. 1992; Eberhard et al. 1993; Rudloff et al. 1994). TIF-IB/SL1 then recruits Pol I and two additional essential factors, TIF-IA and TIF-IC, to the promoter and transcriptional initiation proceeds (Schnapp and Grummt 1991; Schnapp et al. 1993, 1994). The role of the fourth factor, termed UBF, is less well defined. This factor is much more abundant than TIF-IA, TIF-IB, and TIF-IC and appears to activate Pol I transcription in two ways. First, it may stabilize the binding of TIF-IB/SLl to the gene promoter and thus facilitate the assembly of preinitiation complexes. Second, UBF acts as an antirepressor by relieving inhibition exerted by a negative-acting factor that competes for TIF-IB binding to the ribosomal gene promoter (Kuhn and Grummt 1992).

Recently, we purified this repressor protein from Ehrlich ascites tumor cells and found that its biochemical

${ }^{3}$ Corresponding author. properties resemble those of $\mathrm{Ku}$ (Kuhn et al. 1993). Ku, originally identified as an antigen recognized by sera from various autoimmune patients, is a moderately abundant nuclear protein comprising polypeptides of $\sim 70$ and $80 \mathrm{kD}$ in a tightly associated heterodimeric complex (Francoeur et al. 1986; Mimori and Hardin 1986; Yaneva and Busch 1986; Reeves and Sthoeger 1989|. Although some selective interactions with defined promoter elements have been described, most evidence indicates that $\mathrm{Ku}$ binds DNA in an essentially sequence-independent fashion (Mimori and Hardin 1986; de Vries et al. 1989; Paillard and Strauss 1991; Zhang and Yaneva 1992; Blier et al. 1993).

Because of its nuclear location and DNA-binding properties, it has been suggested that $\mathrm{Ku}$ may influence processes such as transcription and replication. Consistent with this, we and others have demonstrated that $\mathrm{Ku}$ is the DNA recognition component of DNA-dependent protein kinase (DNA-PK). In this enzyme, Ku functions to recruit the $\sim 350-\mathrm{kD}$ catalytic component of the $\mathrm{ki}$ nase (p350) to the DNA (Dvir et al. 1993; Gottlieb and Jackson 1993). Because p350 only expresses its catalytic activities when tethered to the template, DNA-PK has the unusual property of only being active when DNA bound. Interestingly, DNA-PK has been found to phosphorylate RNA polymerase II and a variety of sequencespecific regulatory transcription factors (e.g., Jackson et 
al. 1990; Lees-Miller et al. 1990; Anderson and LeesMiller 1992; Peterson et al. 1992; Bannister et al. 1993). Although this suggests that DNA-PK plays a role in transcriptional control, no effect of DNA-PK on transcription has yet been reported.

Our finding that $\mathrm{Ku}$ exerts a negative effect on Pol I-directed rDNA transcription prompted us to investigate whether transcriptional repression is also effected by the DNA-PK holoenzyme. Strikingly, we find that DNA-PK is a much more potent inhibitor of Pol I transcription than is $\mathrm{Ku}$ alone. Furthermore, we show that repression of Pol I transcription by DNA-PK requires ATP hydrolysis and is mediated by phosphorylation of the Pol I transcriptional apparatus. The importance of these findings for the regulation of Pol I transcription in vivo are discussed with reference to recent reports implicating $\mathrm{Ku}$ and DNA-PK in the repair of DNA doublestrand (ds) breaks and in site-specific $V(D) /$ recombination (Finnie et al. 1995; Getts and Stamato 1994; Gottlieb and Jackson 1994; Rathmell and Chu 1994; Taccioli et al. 1994).

\section{Results}

\section{Repression of Pol I transcription by DNA-PK}

Previously, we demonstrated that $\mathrm{Ku}$ associates stably with the rDNA promoter and represses transcription by Pol I, probably through a promoter occlusion mechanism (Kuhn et al. 1993). Because Ku is a component of DNA$\mathrm{PK}$, we investigated whether DNA-PK /consisting of Ku plus p350) also mediates transcriptional repression. To do this, increasing amounts of $\mathrm{Ku}$ or DNA-PK were added to a Pol I transcription system reconstituted with partially purified factors. As shown in Figure 1, both $\mathrm{Ku}$ and DNA-PK evoke a dramatic reduction in Pol I tran-

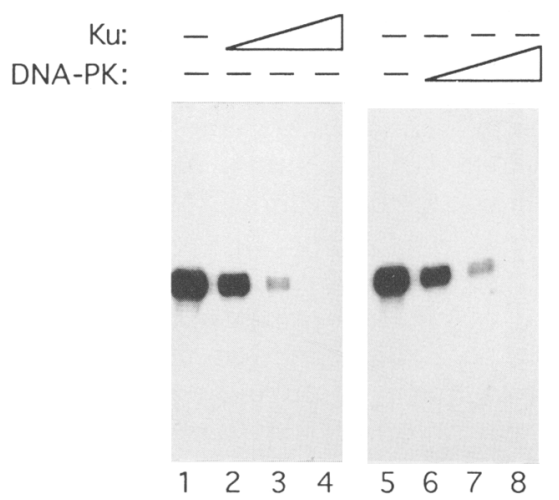

Figure 1. Inhibition of Pol I transcription by DNA-PK. Increasing amounts of $\mathrm{Ku}$ (lanes 1-4) or DNA-PK (lanes 5-8) were preincubated at room temperature with $10 \mathrm{ng}$ of $\mathrm{pMrWT} / \mathrm{Nde}$ I. After 15 min, partially purified Pol I and transcription factors were added and transcription reactions were initiated by addition of ribonucleotides. Reactions 1 and 5 contained no DNA$\mathrm{PK}$ or $\mathrm{Ku}$. Other reactions contained $4.5 \mu \mathrm{l}$ of Ku (lane 2), 6.25 $\mu \mathrm{l}$ of $\mathrm{Ku}$ (lane 3), $9 \mu \mathrm{l}$ of $\mathrm{Ku}$ (lane 4), $0.2 \mu \mathrm{l}$ of DNA-PK (lane 6), $0.5 \mu$ l of DNA-PK (lane 7), or $0.7 \mu l$ of DNA-PK (lane 8). scription. At the highest amounts of $\mathrm{Ku}$ or DNA-PK added, rRNA synthesis is essentially abolished (quantitation reveals that regulation can be $>50$-fold). Interestingly, although the concentration of $\mathrm{Ku}$ polypeptides is similar in the $\mathrm{Ku}$ and DNA.PK preparations, the DNAPK fraction inhibits transcription $\sim 10$-fold more effectively (see legend to Fig. 1). This indicates that inhibition of Pol I transcription occurs much more efficiently in the presence of the DNA-PK catalytic subunit than in its absence.

\section{Transcription inhibition by DNA-PK requires nucleotide hydrolysis}

If p350 is involved in Pol I inhibition, we reasoned that this is likely to be mediated by protein phosphorylation. To test this, we assayed the effect of DNA-PK on transcription under conditions where ATP and GTP were substituted by AMP-PNP and GMP-PNP. These nonhydrolyzable analogs are effective substrates for Pol I but cannot be used by protein kinases. In the presence of these analogs, essentially no DNA-PK-mediated transcriptional inhibition is observed (Fig. 2A, cf. lanes 5-8 with lanes $1-4)$. This indicates that transcriptional repression by DNA-PK requires hydrolysis of the $\beta-\gamma$ bond of ATP and/or GTP and implies that DNA-PK inhibits Pol I transcription by a protein phosphorylation mechanism.

In contrast to the repression of Pol I transcription by DNA-PK, inhibition by high amounts of $\mathrm{Ku}$ occurs equally effectively in the presence of nonhydrolyzable nucleotide analogs (Fig. 2B, cf. lanes 1 and 2 with lanes 5 and 6). This is consistent with previous studies suggesting that high concentrations of Ku inhibit Pol I transcription by binding to the rDNA template and preventing Pol I transcription factors from associating with important cis-acting elements (Kuhn et al. 1993).

As another approach to determine whether ATP hydrolysis by DNA-PK is required for Pol I inhibition, we surveyed several protein kinase inhibitors for their effects. In this way, we discovered that 6-dimethyl amino purine (DMAP) is a potent inhibitor of DNA-PK but does not, on its own, affect Pol I transcription. This inhibition of phosphorylation is observed both with model peptides as substrate (data not shown) and with transcription factor Spl (Fig. 3A). The other proteins labeled in this assay are derived from the kinase preparation, and the most prominent of these corresponds to p350. Strikingly, in the presence of DMAP, DNA-PK no longer inhibits Pol I transcription effectively (Fig. 3B). Therefore, this result provides additional evidence that transcriptional repression by DNA-PK is mediated by protein phosphorylation.

To address the specificity of DNA-PK in terms of inhibiting rRNA synthesis, we tested the effect on Pol I transcription of adding protein kinase A, mitogen-activated protein (MAP) kinase, casein kinase II, or protein kinase C. Importantly, we found that none of these has any effect, even when used at concentrations greater than that required for DNA-PK to elicit total transcrip- 
A

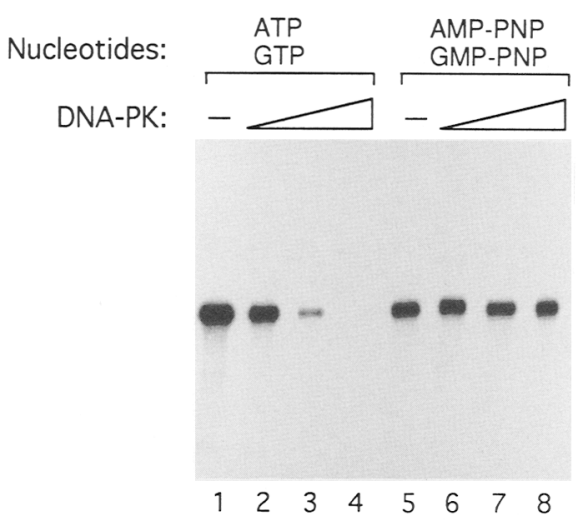

B

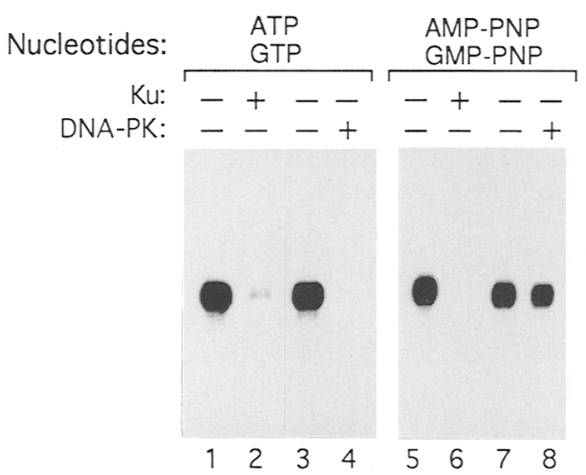

Figure 2. $(A)$ DNA-PK does not inhibit Pol I transcription when ATP and GTP are substituted by nonhydrolyzable analogs. Previous studies have indicated that DNA-PK uses ATP and, to a lesser extent, GTP, but cannot use UTP or CTP (Arias et al. 1991; Peterson et al. 1992). Transcription assays contained $10 \mathrm{ng}$ of $\mathrm{pMrWT} / \mathrm{NdeI}$, Pol I, transcription factors, and increasing amounts of DNA-PK, as indicated. Reactions were performed in the presence of ATP and GTP (lanes 1-4), or with AMP-PNP and GMP-PNP (lanes 5-8). Lanes 1 and 5 contained no DNA-PK lanes 2 and 6 contained $0.05 \mu \mathrm{l}$ of DNA-PK; lanes 3 and 7 contained $0.2 \mu$ lof DNA-PK; and lanes 4 and 8 contained $1 \mu \mathrm{l}$ of DNA-PK. $(B)$ Inhibition of Pol I transcription by high levels of Ku does not require ATP hydrolysis. pMrWT/ NdeI ( $15 \mathrm{ng}$ ) was preincubated for $15 \mathrm{~min}$ with no protein (lanes $1,3,5,7), 5 \mu l$ of $\mathrm{Ku}$ (lanes 2,6), or $0.75 \mu$ l of DNA-PK (lanes 4,8). Pol I and transcription factors were then added, and transcription was initiated by the addition of nucleotides including ATP and GTP (lanes 1-4) or AMP-PNP and GMP-PNP (lanes 5-8).

tion inhibition (data not shown). The inhibitory effect of DNA-PK on Pol I transcription, therefore, appears to be highly specific.

\section{Anti-Ku antisera abrogate inhibition of Pol I transcription by DNA-PK}

Previously, we have demonstrated that various anti-Ku antisera abolish DNA-PK activity (Gottlieb and Jackson 1993). Therefore, we investigated whether an anti-Ku an- tiserum could prevent the inhibition of Pol I transcription by DNA-PK. The specificity of the anti-Ku autoimmune antiserum used in these experiments was first demonstrated in an electrophoretic mobility-shift assay using purified $\mathrm{Ku}$ and a radiolabeled double-stranded DNA (dsDNA) oligonucleotide (Fig. 4A). Addition of this antiserum results in the DNA/Ku complex becoming retarded further in its electrophoretic mobility, indicating that the antibodies recognize Ku but do not prevent DNA binding. When added to in vitro transcription reactions, the anti-Ku antiserum eliminates DNA-PK-me-

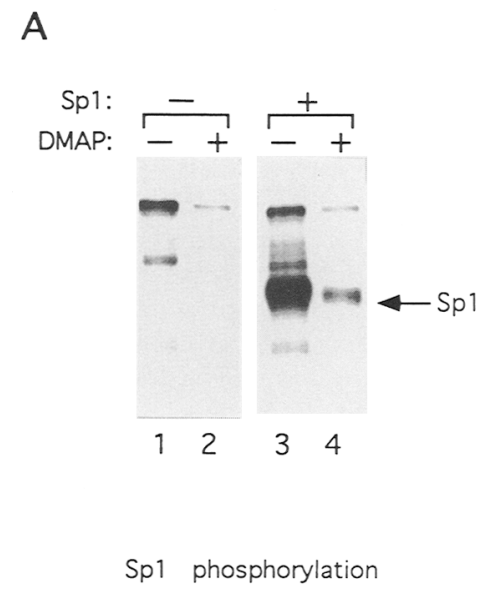

B

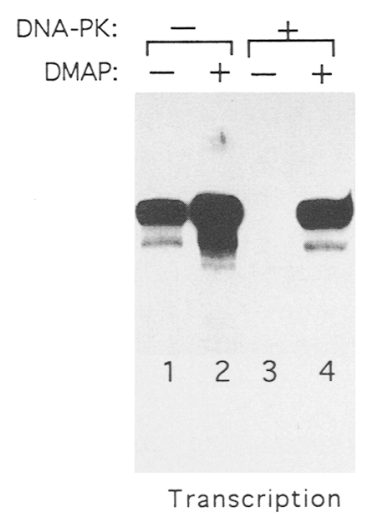

Figure 3. DNA-PK inhibition of Pol I transcription is abrogated by kinase inhibitor DMAP. $\{A \mid \mathrm{Spl}$ phosphorylation assays were conducted with $1.5 \mu \mathrm{l}$ of DNA-PK in the absence (lanes 1,2) or presence (lanes 3,4) of 80 ng of purified Spl. Lanes 1 and 3 lacked DMAP, whereas lanes 2 and 4 contained $2.5 \mathrm{~mm}$ DMAP. Proteins were resolved by electrophoresis on an $8 \%$ SDS-polyacrylamide gel and detected by autoradiography. The location of Spl is indicated. $(B)$ DMAP relieves repression of Pol I transcription by DNA-PK. Transcription assays were conducted using $5 \mu$ l of ascites nuclear extract and $25 \mathrm{ng}$ of $\mathrm{pMrWT} / \mathrm{Ndel}$. DNA and extract were preincubated at $30^{\circ} \mathrm{C}$ with $0.2 \mathrm{mM}$ of ATP in either the absence (lanes 1,2$)$ or presence $($ lanes 3,4$)$ of $1 \mu$ l of DNA-PK. Lanes 1 and 3 lacked DMAP, whereas lanes 2 and 4 contained $2.5 \mathrm{~mm}$ DMAP. After $30 \mathrm{~min}$, transcription was initiated by the addition of the missing ribonucleotides and incubation continued for a further $30 \mathrm{~min}$. 
Figure 4. Inhibition of DNA-PK activity by an anti-Ku antiserum. (A) Electrophoretic mobility-shift assay. Purified $\mathrm{Ku}$ (BioRex 70 fraction; $3 \mu 1$ ) was preincubated for $20 \mathrm{~min}$ at $30^{\circ} \mathrm{C}$ either with control serum (lanes 1-4) or with anti-Ku antiserum (lanes 5-8). Then, 0.1 pmole of a radiolabeled dsDNA oligonucleotide containing the ribosomal core promoter were added and incubation was continued for another $30 \mathrm{~min}$. DNA-protein complexes were analyzed on a $4 \%$ native polyacrylamide gel. Lanes 1 and 5 contained no antiserum; lanes 2 and 6 contained $0.03 \mu \mathrm{l}$ of antiserum; lanes 3 and 7 contained $0.15 \mu \mathrm{l}$ of antiserum; lanes 4 and 8 contained $0.75 \mu \mathrm{l}$ of antiserum. The electrophoretic positions of free DNA, the Ku/DNA complex, and the antibody/Ku/DNA complex are indicated. $(B)$ Effect of anti-Ku antiserum on DNA-PK-mediated inhibition of Pol I transcription. Control serum $(0.15 \mu \mathrm{l})$ (lanes 1,2) or anti-Ku antiserum (lanes 3,4) was preincubated for $15 \mathrm{~min}$ at room temperature with Pol I and transcription initiation factors TIF-IA, TIF-IB, TIF-IC, and UBF. Preincubations were performed in the absence (lanes 1,3) or presence (lanes 2,4) of $0.75 \mu$ l of DNA-PK. After addition of 10 ng of $\mathrm{pMrWT} / \mathrm{NdeI}$, incubations were continued for another $15 \mathrm{~min}$ before transcription was initiated by the addition of ribonucleotides. diated repression of rRNA synthesis, whereas a control antiserum that does not recognize Ku has no effect (Fig. 4B). Similar abolition of DNA-PK repression is also obtained with an anti-Ku monoclonal antibody that inhibits the binding of $\mathrm{Ku}$ to DNA (data not shown). These data confirm that Ku plays an important role in the inhibition of Pol I transcription by DNA-PK. We have shown previously by UV protein/DNA cross-linking that $\mathrm{p} 350$ is targeted to DNA by association with $\mathrm{Ku}$ and that anti-Ku autoimmune antisera block the $\mathrm{Ku}-\mathrm{p} 350$ interaction (Gottlieb and Jackson 1993). Therefore, the abolition of Pol I transcriptional repression by the anti$\mathrm{Ku}$ antiserum provides additional evidence that $\mathrm{p} 350 \mathrm{ex}$ ecutes an important function in this process.

\section{Transcriptional repression requires DNA ends}

It has been established that DNA-PK activation requires DNA double-strand breaks and does not occur with closed circular DNA molecules (Carter et al. 1990; Gottlieb and Jackson 1993). Therefore, we compared the effects of DNA-PK on transcription from both linear and circular rDNA templates. In these experiments, an rDNA minigene construct pMrC552-650 was used in which a fragment containing a Pol I-specific transcription terminator is inserted downstream of the promoter (Fig. 5A). In the reconstituted transcription system, which contains limiting amounts of transcription termination factor TTF-I, about half of the transcripts synthesized terminate specifically at the terminator. Therefore, on a linearized template, two types of RNA are produced: one corresponding to terminated transcripts, and the other corresponding to readthrough transcripts that terminate at the EcoRI site at the end of the DNA mol ecule. On the uncut template, however, long readthrough transcripts are observed at the top of the gel in addition to the terminated transcripts. In the case of the linear template, we found that DNA-PK causes a dramatic inhibition of both terminated and readthrough transcription (Fig. 5B, lanes 1-3). In contrast, no effect is observed on transcription from the closed circular DNA (lanes 4-6). These results are therefore consistent with transcriptional inhibition by DNA-PK requiring free DNA ends.

\section{DNA-PK and the Pol I transcription complex must be colocalized on the same DNA molecule}

An important question concerning the mechanism of transcriptional inhibition by DNA-PK is whether the kinase must be bound to the same template as its target transcription complexes or whether it can function in trans from another DNA molecule. To address this issue, transcription reactions were performed in the presence of increasing amounts of a synthetic double-strand oligonucleotide as a nonspecific competitor, the rationale being that oligonucleotide addition would titrate DNA-PK away from the rDNA template. As shown in Figure 6A, oligonucleotide addition abolishes the ability of DNA-PK to inhibit Pol I transcription (lanes 4-6). In the absence of DNA-PK, however, the oligonucleotide has a minimal effect (lanes 1-3). Significantly, oligonucleotide addition does not inhibit the inherent catalytic activity of DNA-PK, as demonstrated by peptide phosphorylation experiments (Gottlieb and Jackson 1993). If DNA-PK only functions in cis, one would predict that it would work most efficiently at low template concentra- 
A

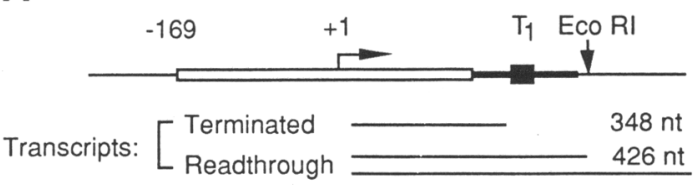

transcriptional initiation site. Although a DNA looping pathway cannot be excluded, we speculate that DNA$\mathrm{PK} / \mathrm{Ku}$ gains access to the Pol I transcriptional machinery by a DNA-scanning mechanism.

A B

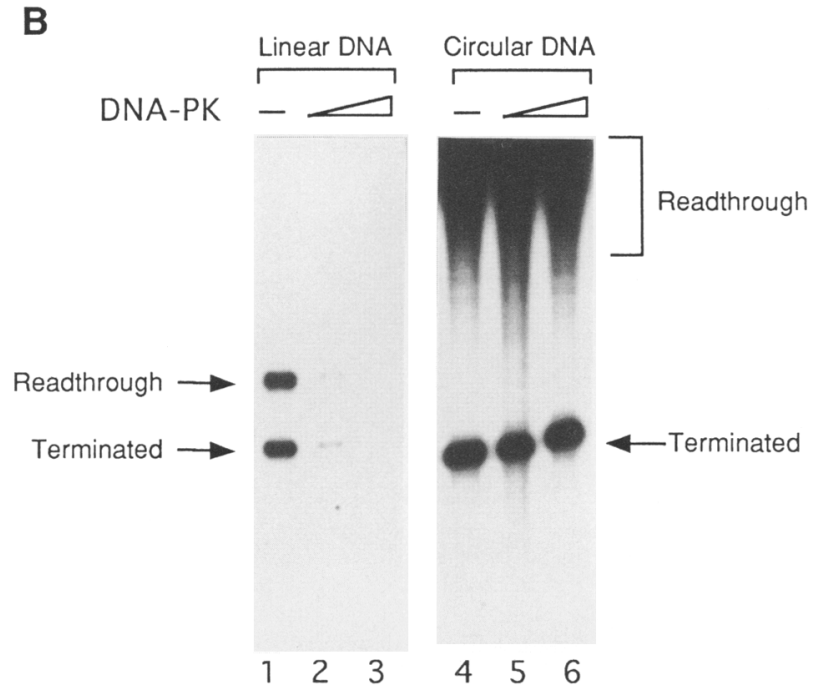

Figure 5. Inhibition of Pol I transcription by DNA-PK requires DNA ends. $(A)$ Schematic representation of the rDNA minigene construct pMrC552-650. The open bar represents sequences from the $5^{\prime}$ region of the murine rDNA repeat, including 167 nucleotides derived from the CAT gene. The black box represents the 18-bp Sal box terminator element flanked by 3 '-terminal sequences extending to positions +552 and +650 with respect to the end of the $28 \mathrm{~S}$ rRNA (solid bar). $(B)$ Transcription reactions were performed in the presence of $10 \mathrm{ng}$ of the minigene pMrC552-650. The template was either linearized with EcoRI (lanes 1-3) or was used in the uncut circular form (lanes 4-6). Reactions contained no DNA-PK (lanes 1,4), $0.1 \mu l$ of DNA-PK (lanes 2,5), or $1 \mu$ l of DNA-PK (lanes 3,6). The positions of terminated and readthrough transcripts are indicated.

tions, where all DNA molecules contain bound kinase. Indeed, we find that a given amount of DNA-PK inhibits Pol I transcription strongly when $5 \mathrm{ng}$ of rDNA is used but only weakly with $25 \mathrm{ng}$ of template (Fig. 6B).

In all of the above studies, the rRNA promoter was positioned close to the end of the linear DNA molecule. Although DNA ends are required for the initial binding of Ku/DNA-PK to DNA, previous reports have suggested that once bound to the template, it can then translocate to internal positions on the DNA molecule (de Vries et al. 1989; Paillard and Strauss 1991; Gottlieb and Jackson 1993|. We were thus prompted to test whether transcriptional inhibition was affected by the location of the DNA ends in regard to the transcription complex. As shown in Figure 6C, DNA-PK is equally effective at inhibiting Pol I transcription when the DNA end is close upstream, close downstream, or $>1500$ bp away from the

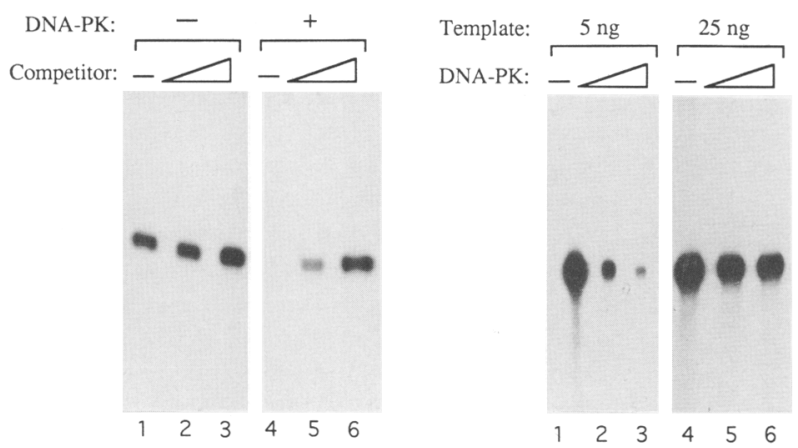

C

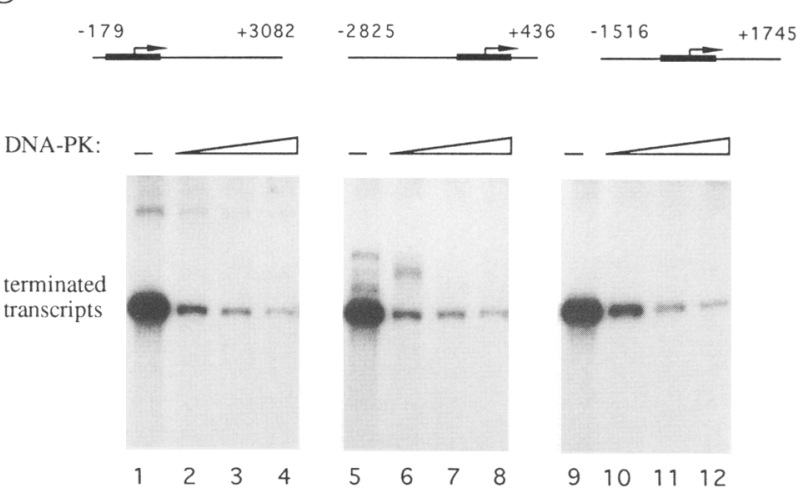

Figure 6. DNA-PK must bind to the same template molecule as the Pol I transcription complex to repress rRNA synthesis but functions equally effectively from proximal and distal DNA ends. (A) Oligonucleotide competition assays. pMrWT/NdeI (10 ng) was transcribed in the absence (lanes 1-3) or presence (lanes 4-6) of $0.5 \mu \mathrm{l}$ of DNA-PK. Reactions were conducted in the absence of oligonucleotide (lanes 1,4 ) or contained 0.25 pmole (lanes 2,5) or 0.75 pmole (lanes 3,6) of a DNA oligonucleotide containing the octamer target sequence. (B) DNA-PK only works effectively at low template concentrations. Transcription assays were conducted as in $A$ in the absence of DNA-PK (lanes 1,4 ), or in the presence of $0.2 \mu \mathrm{l}$ (lanes 2,5) or $0.4 \mu$ l (lanes 3,6) of DNA-PK. Lanes 1-3 and lanes 4-6 contained $5 \mathrm{ng}$ and $25 \mathrm{ng}$ of the template pMrWT/Ndel, respectively. $(C)$ Varying the distance between DNA ends and the rDNA promoter does not affect DNA-PK-mediated transcriptional inhibition. The minigene construct pMrC552-650 (10 ng) was linearized with $P_{s t I}$ (lanes 1-4), EcoRI (lanes 5-8), or Cfrl0 I (lanes 9-12) and transcribed in the presence of recombinant transcription termination factor TTFI to yield 348-nucleotide terminated transcripts. Reactions contained no DNA-PK (lanes 1,5,9), $0.5 \mu \mathrm{l}$ of DNAPK (lanes 2,6,10), $0.75 \mu$ l of DNA-PK (lanes 3,7,11), or $1 \mu$ of DNA-PK (lanes 4,8,12). The position of the rDNA insert (bold line) within the linearized plasmid fragments is indicated above. The numbers at the ends correspond to the numbers of nucleotides that the ends are upstream $(-)$ or downstream $|+|$ of the initiation site. 
Figure 7. DNA-PK can function after preinitiation complex formation. $(A)$ Single-round transcription assays. Pol I and transcription initiation factors were preincubated with $10 \mathrm{ng}$ of template $\mathrm{pMrWT} / \mathrm{NdeI}$ at room temperature. After $15 \mathrm{~min}, 0.75 \mu \mathrm{l}$ of DNA-PK was added (lanes 2,4) and incubations were continued for an additional 15 min before transcription was initiated by the addition of ribonucleotides; reactions were then conducted for $30 \mathrm{~min}$ at $30^{\circ} \mathrm{C}$. To prevent reinitiation of transcription in lanes 3 and 4 , heparin was added to a final concentration of $15 \mu \mathrm{g} / \mathrm{ml}$ to the preinitiation complexes assembled with DNA-PK together with ribonucleotides. $(B)$ Runoff and trimer transcription assays. pMrWT/NdeI (30 ng; lanes 1-6) or $\mathrm{pB}$ $\mathrm{SSK}^{+} / E \operatorname{ECORI}(30 \mathrm{ng}$; lanes 7,8 ) was preincubated with Pol I and transcription factors at $30^{\circ} \mathrm{C}$ in a $50-\mu l$ reaction containing 0.5 $\mathrm{mM}$ each of ATP and CTP. Preincubations were performed without (lanes 1,2,5-8) or with (lanes 3,4) $2.5 \mathrm{~mm}$ DMAP. After 30 min, $1.5 \mu$ l of DNA-PK were added to lanes $2,4,6$, and 8 , and incubations were continued for another $15 \mathrm{~min}$. Reactions were then divided into two equal portions; one was used to synthesize runoff transcripts by the addition of $0.5 \mathrm{~mm}$ GTP, $10 \mu \mathrm{M}$ $\mathrm{UTP}$, and $2 \mu \mathrm{Ci}$ of $\left[\alpha^{-32} \mathrm{P}\right] \mathrm{UTP}$; the other was used to synthesize ACU trimers by the addition of $5 \mu \mathrm{Ci}$ of $\left[\alpha^{-{ }^{3}}\right.$ P $\}$ UTP alone. Runoff and trimer transcription products are indicated by arrows in the upper and lower panels, respectively. Lanes 5 and 6 generated no runoff or trimer transcripts. Because these reactions contained Pol I but no other Pol I factors, this demonstrates that transcription is dependent on these other factors. Lanes 7 and 8 , similarly, produced no products. Because these reactions contained vector DNA lacking the rDNA sequences, this shows that transcription observed in lanes $1-4$ is Pol I promoter dependent.

\section{DNA-PK functions in single-round Pol I transcription assays}

The repression of transcription by DNA-PK could potentially involve inhibition of preinitiation complex formation, transcription initiation, and/or transcription elongation. As a first step toward identifying the mechanism of DNA-PK action, we performed single-round transcription experiments. In these assays, transcription preinitiation complexes were assembled on the rDNA promoter by preincubating the template with partially purified transcription factors TIF-IA, TIF-IB, TIF-IC, UBF, and Pol I. After complex formation, DNA-PK was then added and transcription was initiated by the addition of ribonucleotides. Reactions were performed in the absence (Fig. 7A, lanes 1,2$)$ or in the presence (lanes 3,4$)$ of $15 \mu \mathrm{g} / \mathrm{ml}$ of heparin. At this concentration of heparin, preformed Pol I preinitiation complexes are not disrupted, but assembly of new complexes and reinitiation is prevented (Hawley and Roeder 1985; A. Kuhn, unpubl.). Clearly, inhibition of transcription is still observed when DNA-PK is added after the formation of fully assembled complexes in both multiple- and single-round transcription reactions. This indicates that DNA-PK can act at a step after preinitiation complex assembly, presumably by disrupting such complexes or by blocking either transcriptional initiation or elongation.

\section{DNA-PK inhibits abortive initiation by Pol I}

In an attempt to pinpoint the steps at which DNA-PK
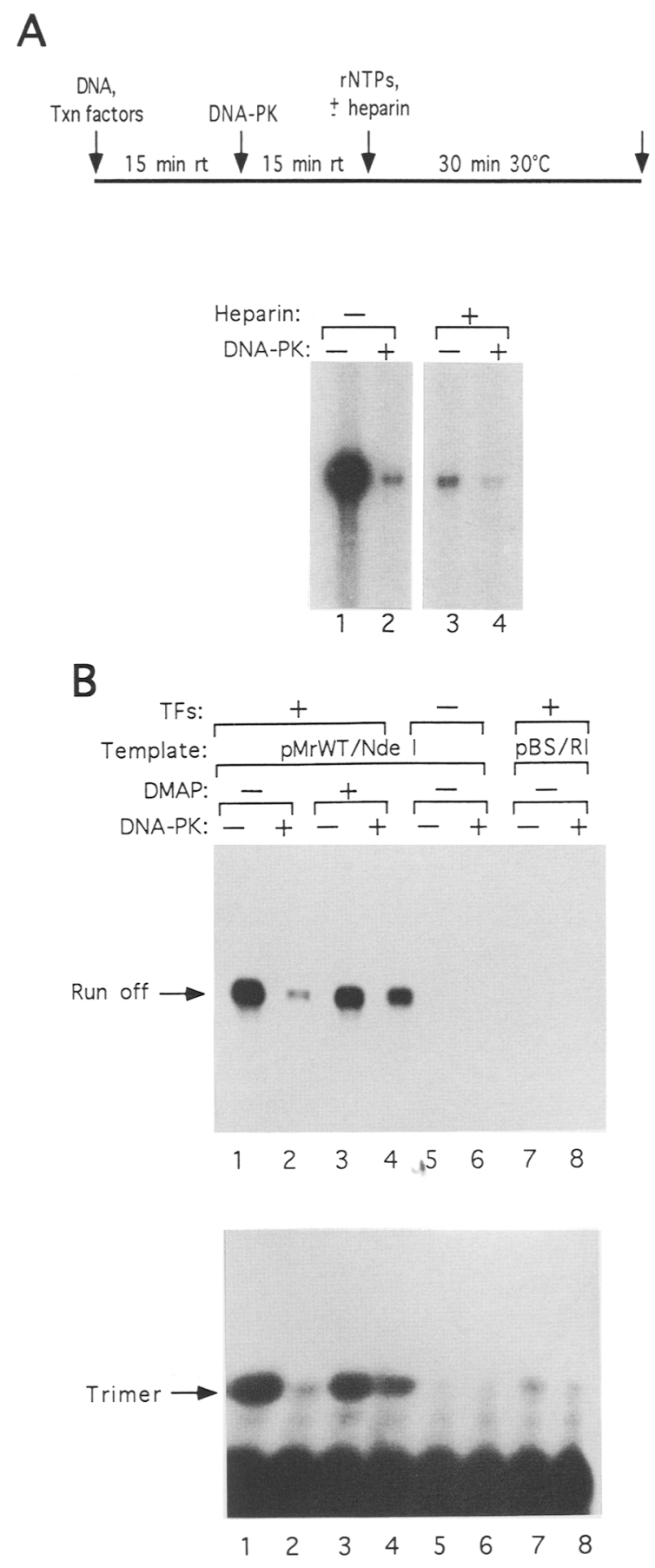

exerts its inhibitory effects, abortive initiation assays were performed. In these experiments, partially purified Pol I and transcription factors were incubated with the DNA template in the presence of ATP plus CTP / the first two nucleotides of the mouse pre-rRNA) to form initiated complexes. DNA-PK was then added, and the reaction was divided into two parts. One-half was supplemented with GTP and labeled UTP, and transcription was allowed to proceed for $30 \mathrm{~min}$. The other half was supplemented with labeled UTP alone and was incu- 
bated for $15 \mathrm{~min}$. In the latter reactions, Pol I catalyzes the incorporation of the third nucleotide into the rRNA. However, as the subsequent ribonucleotide used in rRNA synthesis (GTP) is not present, Pol I cannot progress further into the elongation phase of transcription. Instead, it releases the trinucleotide pppApCpU from the template and, without leaving the ternary transcription complex, begins transcription again (McClure 1980). In this assay, therefore, abortive transcriptional initiation but not elongation takes place. Significantly, we find that DNA-PK inhibits both full-length runoff transcription (Fig. 7B, top) and trimer synthesis (bottom). This result indicates that DNA-PK represses transcriptional initiation or promoter clearance, or both.

\section{DNA-PK targets components of the Pol I transcriptional machinery}

The above results suggest that DNA-PK represses Pol I transcription by phosphorylating a component of the Pol I transcriptional apparatus. One possibility is that DNAPK inhibits transcription by phosphorylating Pol I and thus decreases or abolishes its catalytic activity. To determine whether this is the case, we used "tailed template" assays in which Pol I initiates transcription in a nonspecific fashion that is independent of other Pol I general transcription factors (Kuhn et al. 1990). When DNA-PK was used in such assays, it was found to have no effect (Fig. 8). Importantly, these experiments were conducted under essentially the same conditions as those used for specific transcription assays, where DNAPK inhibits transcription strongly. Moreover, peptide phosphorylation assays indicate that DNA-PK is highly active in the tailed template transcription reactions /data not shown). Taken together with other data, these results indicate that DNA-PK represses specific factor-dependent transcription by Pol I but does not affect factorindependent initiation or transcriptional elongation.

To identify the relevant target for DNA-PK in the Pol I transcription apparatus, we performed experiments in which DNA-PK alone, or DNA-PK in combination with individual Pol I initiation factors, were preincubated either in the presence or absence of ATP (Fig. 9). To prevent dephosphorylation of target proteins occurring during subsequent incubation steps, the ATP analog ATP $\gamma$ S was employed. Next, DMAP was added to inhibit DNAPK activity, and transcription was initiated by the addition of remaining factors and nucleotides. When DNA$\mathrm{PK}$ alone is present in the preincubation reaction, no transcriptional inhibition is observed (lanes 1,2). This finding demonstrates that autophosphorylation of DNA$\mathrm{PK}$ is not involved in the repression of transcription. Similarly, no inhibition of transcription occurs when DNA-PK is preincubated with UBF (lanes 5,6), revealing that UBF is not a relevant DNA-PK target. In contrast, preincubation with TIF-IB results in a reproducible inhibition of transcription (lanes 3,4 ). This result suggests that DNA-PK targets either the TBP-containing TIF-IB factor itself or another component present in the partially purified TIF-IB fraction. Significantly, the DNA-PK

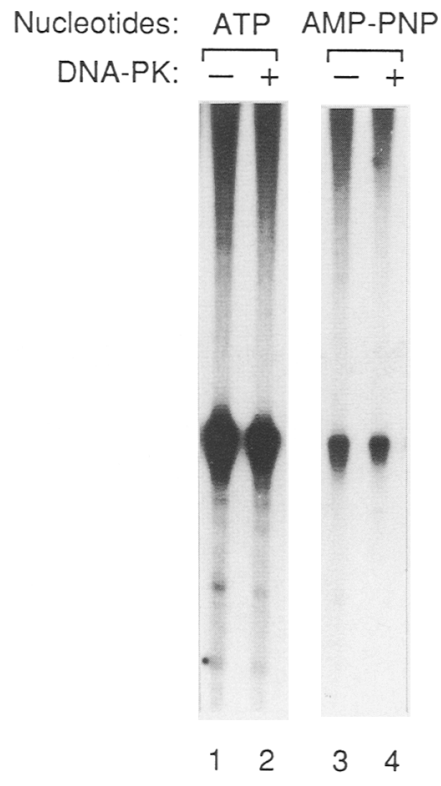

Figure 8. DNA-PK does not inhibit transcription elongation. Tailed template pCAT552.650/Smal (20 ng) was transcribed with $7.5 \mu \mathrm{l}$ of Pol I in the absence of DNA-PK (lanes 1,3) or presence of $1.5 \mu$ l of DNA-PK (lanes 2,4). Reactions contained $200 \mu \mathrm{g} / \mathrm{ml}$ of $\alpha$-amanitin, $0.5 \mathrm{mM}$ of the dinucleotide UpG / to prime unspecific transcription of Pol I), and either ATP (lanes 1,2) or AMP-PNP (lanes 3,4). Note that the relationship between the amount of DNA-PK and the amount of tailed template was the same as that used in specific transcription assays. Peptide phosphorylation studies indicate that DNA-PK is active in tailed template reactions (data not shown).

inhibitory effect increases when the preincubation reactions not only contain TIF-IB but also UBF, Pol I, or a combination of all factors (lanes 7-12). One explanation for these results is that the formation of higher order complexes renders the target proteins more accessible to DNA-PK.

An important question regarding the molecular mechanism of DNA-PK-mediated transcriptional inhibition is whether phosphorylation of the transcriptional machinery leads to the disruption of the initiation complex or whether inactive complexes remain associated with the rDNA promoter. To address this question, we performed rescue experiments (Fig. 10). In these studies, transcription complexes were first phosphorylated by DNA-PK. Next, each of the chromatographic fractions needed for transcription was added back to separate reactions, together with DMAP to ensure that DNA-PK would not phosphorylate the added factors. Addition of the individual factors to control reactions lacking DNA-PK does not increase transcription significantly, indicating that saturating amounts of each of the Pol I factors were used to assemble the preinitiation complexes (lanes 1-6). Interestingly, DNA-PK-mediated transcriptional inhibition cannot be rescued by the addition of either TIF-IB (lane 8), UBF (lane 9), Pol I (lane 10), or TIF-IA/TIF-IC (lane 11). Even supplementing the reaction with all factors 

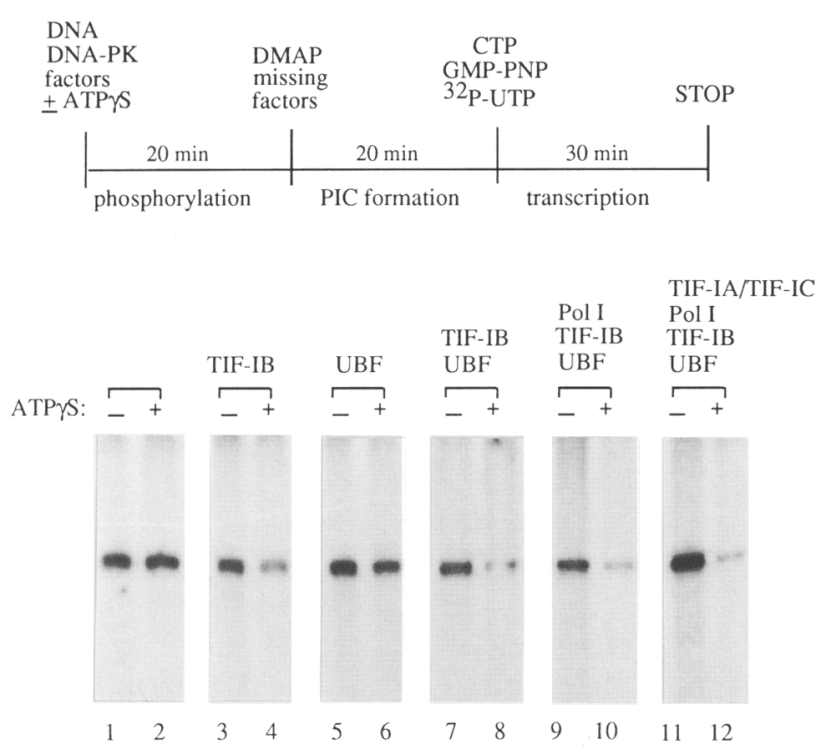

Figure 9. Phosphorylation of the TIF-IB fraction by DNA-PK decreases transcription. Reactions containing $20 \mathrm{ng}$ of template DNA, $1.5 \mu \mathrm{l}$ of DNA-PK, and the factors indicated above the lanes were preincubated in the absence of ATP $\gamma \mathrm{S}$ or in the presence of $0.2 \mathrm{mM}$ of ATP $\gamma$ S. After $20 \mathrm{~min}$, DNA-PK was inhibited by addition of $2.5 \mathrm{mM}$ DMAP, and the assays were complemented with the missing factors. ATP $\gamma \mathrm{S}(0.2 \mathrm{mM})$ was added to the ATP-free reactions and incubation was continued for another $20 \mathrm{~min}$ to allow the assembly of initiation complexes. Transcription was started by adding the missing nucleotides.

together fails to restore transcription (lane 12). These results suggest that the protein that is inactivated by DNA-PK remains bound to the promoter and cannot be replaced effectively by untreated factors added subsequently.

\section{Discussion}

We have demonstrated that DNA-PK, comprising Ku plus p350, functions as a powerful inhibitor of Pol I transcription. Because we had shown previously that $\mathrm{Ku}$ alone also represses transcription and that this is mediated by template occlusion, it was important to establish whether inhibition by DNA-PK occurs solely through this route or whether p350 is also involved. Several lines of evidence indicate that p350 is involved in transcriptional repression and that its effects are mediated by phosphorylation. First, DNA-PK inhibits transcription $\sim 10$-fold more effectively than $\mathrm{Ku}$ alone. Second, inhibition of transcription by DNA-PK does not occur in the presence of nucleotide analogs AMP-PNP and GMPPNP, indicating that it requires nucleotide hydrolysis. Third, antibodies against Ku that do not inhibit Ku binding to DNA but interfere with p350 recruitment, abrogate transcriptional repression by DNA-PK. Fourth, Pol I transcription inhibition by DNA-PK does not occur in the presence of DMAP, which inhibits kinase activity. In contrast, inhibition by high amounts of Ku still occurs in the presence of DMAP, and with AMP-PNP and GMPPNP.

If the inhibition of Pol I transcription by purified DNA-PK is of significance, one would expect that DNAPK present in crude cell extracts would serve as an endogenous transcriptional repressor. In line with this, we attain higher levels of transcription using circular templates than with linear templates (see Fig. 5). Moreover, we find that DMAP addition to crude murine transcription extracts leads to increased Pol I transcription (e.g., Fig. 3B, cf. lanes 1 and 2). This indicates that an endogenous kinase in murine extracts represses transcription by Pol I. When DMAP is added to HeLa cell extracts, even larger stimulations of Pol I transcription are obtained (data not shown), consistent with the fact that human cells contain much more DNA-PK than mouse cells (Anderson and Lees-Miller 1992; Finnie et al. 1995). Furthermore, in line with the fact that DNA-PK functions in cis and therefore operates efficiently only at low template concentrations, we find that the addition of DMAP to HeLa cell extracts stimulates transcription effectively only when low amounts of template are used. Although we cannot exclude the possibility that transcriptional activation by DMAP is through it inhibiting some other negatively acting kinase, these data suggest strongly that endogenous DNA-PK in crude mammalian cell extracts represses Pol I transcription. Interestingly, Labhart (1994) has reported recently that a DMAP-sensitive inhibitor of Pol I transcription exists in Xenopus and that this inhibitor acts after preinitiation complex

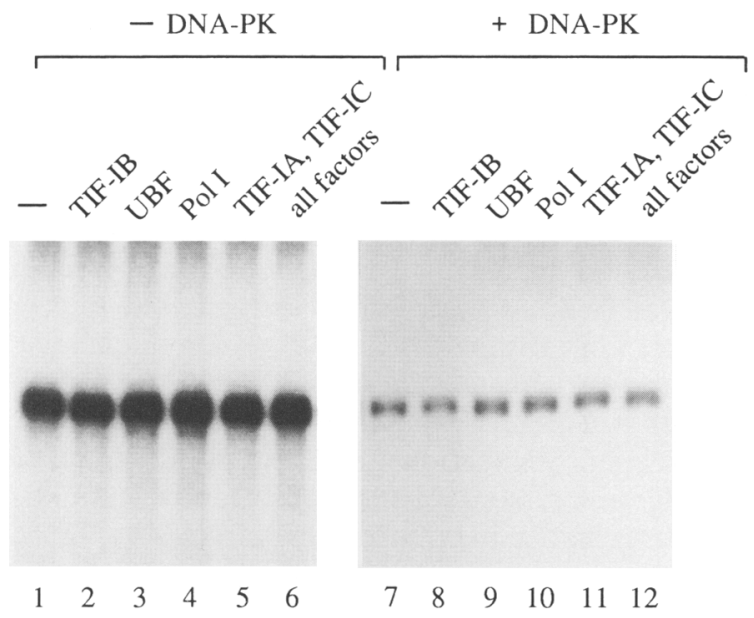

Figure 10. Transcriptional inhibition is not rescued by individual transcription factors. Template DNA (15 ng of pMrWT/ NdeI) and saturating amounts of TIF-IB, UBF, Pol I, and TIF-IA/ TIF-IC were incubated in the presence of $0.2 \mathrm{~mm}$ of ATP $\gamma \mathrm{S}$ either without DNA-PK (lanes 1-6) or with $1 \mu$ l of DNA-PK (lanes 7-12). After 30 min, DNA-PK activity was inhibited by the addition of $2.5 \mathrm{mM}$ of DMAP, and reactions were supplemented with additional TIF-IB (lanes 2,8), UBF (lanes 3,9), Pol I (lanes 4,10), TIF-IA/TIF-IC (lanes 5,11), or with a combination of all factors (lanes 6,12). Transcription reactions were initiated by the addition of CTP, GMP-PNP, and $\left[{ }^{32} \mathrm{P}\right] \mathrm{UTP}$ and allowed to proceed for $30 \mathrm{~min}$. 
formation. Because of the strong parallels with our findings, and in light of the fact that DNA-PK is present in Xenopus (Anderson and Lees-Miller 1992; Finnie et al. 1995), it is tempting to speculate that the endogenous Pol I inhibitor identified by Labhart (1994) is Xenopus DNA-PK.

To determine the steps in Pol I transcription at which DNA-PK functions, we performed single-round transcription experiments, abortive initiation assays, and factor-independent transcription of a tailed template. We find that DNA-PK still represses transcription in singleround transcription assays, demonstrating that it can either disrupt preformed transcription complexes or inhibit a later stage of the transcription process. To see whether DNA-PK inhibits the formation of the first phosphodiester bonds of rRNA, we measured its effects on the synthesis of abortive trimer transcripts. These studies revealed that DNA-PK inhibits trinucleotide formation efficiently, suggesting that DNA-PK represses transcriptional initiation or promoter clearance, or both. In contrast with its effects in other assays, DNA-PK is unable to repress Pol I transcription in tailed template experiments, where transcriptional initiation occurs in a nonsequence-specific fashion that is independent of auxillary transcription factors. These results suggest that the transcriptional elongation activity of Pol I is not affected by DNA-PK-mediated phosphorylation.

As an approach to define relevant targets for DNA-PK, we prephosphorylated Pol I and the individual initiation factors and then compared their activities with untreated controls. These studies indicated that the activity of TIF-IB, the TBP-containing factor that confers promoter selectivity to Pol I, is inhibited by DNA-PK. However, the presence of Pol I and the two Pol I-associated factors TIF-IA and TIF-IC augments transcriptional repression. These observations can be interpreted in two ways. One is that transcriptional inhibition is mediated by proteins that are present as a contaminant in the partially purified fractions and that this putative factor can only repress transcription when phosphorylated by DNA-PK. The other explanation, which we currently favor, is that DNA-PK inhibits TIF-IB by targeting one or more subunits of this multiprotein complex. In this case, the enhanced inhibition caused by Pol I and the TIF-IA/TIF-IC fraction might be attributed to these factors stabilizing the interaction of TIF-IB with the template or by them inducing a conformational alteration in TIF-IB that facilitates recognition by DNA-PK. The suggestion that TIF-IB may be the DNA-PK target is supported by the fact that transcriptional inhibition cannot be overcome by the subsequent addition of any or all of the transcription factor fractions, implying that the inactivated target protein remains bound to the template and prevents the assembly of productive initiation complexes.

An important question regarding DNA-PK is whether it can access the Pol I transcriptional machinery within the cell. Previous studies have shown that $\mathrm{Ku}$ exists within the nucleolus and nuclear cortex (Higashiura et al. 1992) and have revealed that nucleolar localization varies throughout the cell cycle (Yaneva and Jhiang 1991). When we analyzed the localization of $\mathrm{Ku}$ and p350, we found that both can be detected in the nucleoplasm and in nucleoli, indicating that DNA-PK is likely to be able to access the Pol I transcriptional apparatus in vivo (data not shown). We found, however, that nucleolar staining varies considerably from cell to cell; in some, nucleoli stain intensely, whereas in others, essentially no nucleolar fluorescence can be detected for either Ku or p350. Interestingly, cell synchronization experiments that we have performed reveal that both $\mathrm{Ku}$ and p350 are present in the nucleoli of $G_{2}$ phase cells, but neither are detectable in the nucleoli of S-phase cells. Although the physiological consequences of such changes in localization are unclear, it is tempting to speculate that changes in DNA-PK distribution might mediate cell cycle variations in rRNA synthesis, or might reflect cell cycle-dependent differences in the ability of the cell to modulate transcription in response to DNA damage (see below).

The finding that DNA-PK activation requires DNA ends or other discontinuities in the DNA double helix (Gottlieb and Jackson 1993; Morozov et al. 1994) has suggested that DNA-PK might be involved in recombination and/or DNA repair. Indeed, it has been shown recently that Ku DNA-binding function and DNA-PK activity are absent in the cell line $x r s-6$ that is defective in DNA double-strand break repair and $V(D) /$ recombination (Finnie et al. 1995; Getts and Stamato 1994; Gottlieb and Jackson 1994; Rathmell and Chu 1994; Taccioli et al. 1994). Furthermore, we have established that Ku80 expression complements the defects of xrs-6 cells and have concluded that the $80-\mathrm{kD}$ subunit of $\mathrm{Ku}$ is encoded by the gene XRCC5 (Taccioli et al. 1994). On this basis, we speculate that the inhibition of Pol I transcription by DNA-PK may be an important response to dsDNA breaks generated within or in the vicinity of the rDNA. Specifically, we propose that DNA-PK plays two distinct roles in ensuring the efficient repair of double-strand breaks in rRNA transcription units. First, it might act to inhibit the very high transcription across this region that would otherwise interfere with the assembly and functioning of the DNA repair apparatus. Second, DNA-PK might function in repair itself, either directly or by recruiting or activating components of the repair machinery. DNA-PK may also perform analogous roles at sites of recombination. Finally, it is possible that DNA-PK plays similar roles at DNA breaks in Pol II and Pol III transcription units, although the effect of DNA-PK Pol II and Pol III transcription remains to be determined.

\section{Materials and methods}

\section{Plasmid constructs}

The minigene construct pMrC552-650 contains murine $5^{\prime}$ terminal ribosomal sequences from -169 to +155 , a 167-bp StuIPvull fragment from the chloramphenicol acetyltransferase (CAT) gene and a 3' terminal rDNA spacer fragment spanning sequences +552 to +650 with respect to the $3^{\prime}$ end of the $28 \mathrm{~S}$ rRNA-coding region. The tailed template pCAT554-650 contains 151 nucleotides from the bacterial CAT gene fused to a 
97-bp fragment derived from the 3 -terminal murine rDNA spacer. Synthesis of the single-stranded 3' extension by ligation of the oligonucleotide 5'-GATCAAAAAAACCA-3' to the Bg/II site of pCAT554-650 has been described previously (Kuhn et al. 1990|. pMrWT contains a 324-bp 5' terminal SalI-SmaI fragment (from -169 to +155 ), including the murine ribosomal gene promoter.

\section{Purification of DNA-PK, Ku, transcription factors, and Pol I}

Cultivation of Ehrlich ascites cells, extract preparation, and fractionation of individual transcription factors has been described (Schnapp and Grummt 1991). Briefly, a mixture of nuclear and cytoplasmic extracts was chromatographed on DEAESepharose CL-6B followed by fractionation on heparin-Ultrogel A4-R. Bound proteins were step-eluted with 200,400,600, and $1000 \mathrm{mM} \mathrm{KCl}$ in buffer AM $[20 \mathrm{~mm}$ Tris- $\mathrm{HCl}(\mathrm{pH} 7.91,0.1 \mathrm{~mm}$ EDTA, $20 \%$ glycerol, $5 \mathrm{~mm} \mathrm{MgCl}_{2}$ ]. The $200 \mathrm{~mm}$ fraction $(\mathrm{H}$ 200) contained TIF-IA and TIF-IC, the $\mathrm{H} 400$ fraction contained Pol I, the H-600 fraction contained TIF-IB, and the H-1000 fraction contained upstream binding factor (UBF). Further purification of Pol I involved fractionation on a Mono $S$ column followed by chromatography on Mono Q. TIF-IA and TIF-IC were fractionated on Q-Sepharose and by chromatography on Mono Q. TIF-IB was purified on CM-Sepharose and Mono S (Eberhard et al. 1993). UBF was purified from $\mathrm{H}-1000$ fractions by chromatography on Mono S, Mono Q, and Bio-Rex 70. Although each of the factors was only purified partially, they were not cross-contaminated with any of the other factors required for specific transcription in vitro. $\mathrm{Ku}$ was purified as previously (Kuhn et al. 1993), and DNA-PK was isolated from crude wheat germ agglutinin-depleted HeLa nuclear extract (Jackson and Tjian 1989) by the method of Lees-Miller et al. (1990). Spl phosphorylation assays were performed as described previously (Jackson et al. 1990; Gottlieb and Jackson 1993).

\section{In vitro transcription assays}

The template pMrWT was linearized with NdeI to generate transcripts of 371 nucleotides. The terminator-containing template pMrC552-650 was used either in the circular form yielding 348-nucleotide terminated transcripts or was linearized to obtain defined readthrough transcripts of 426 nucleotides. The tailed template pCAT554-650 was digested with SmaI to generate runoff transcripts of 290 nucleotides. Transcription reactions were performed in 25- $\mu$ l assays containing 10-30 ng of template DNA and $15 \mu \mathrm{l}$ of a mixture of Pol I and transcription factors. Reactions contained $12 \mathrm{~mm}$ Tris- $\mathrm{HCl}(\mathrm{pH} 7.9), 85 \mathrm{~mm}$ $\mathrm{KCl}, 5 \mathrm{~mm} \mathrm{MgCl}, 10 \mathrm{~mm}$ creatine phosphate, $10 \%$ glycerol, $0.12 \mathrm{~mm}$ EDTA, $0.5 \mathrm{~mm}$ dithioerythritol, $0.5 \mathrm{~mm}$ each of ATP, CTP and GTP, $12.5 \mu \mathrm{M}$ UTP, and $1-2 \mu \mathrm{Ci}$ of $\left[\alpha^{-{ }^{32}} \mathrm{P}\right] \mathrm{UTP}$. Where indicated, ATP and GTP were replaced by AMP-PNP or GMPPNP. Reactions were incubated at $30^{\circ} \mathrm{C}$ for $\mathrm{l} \mathrm{hr}$ and were then processed for gel analysis.

\section{Abortive initiation assay}

Pol I and transcription factors were preincubated with the DNA in the presence of $0.5 \mathrm{~mm}$ each of ATP and CTP to form initiated complexes. After $30 \mathrm{~min}$, DNA-PK was added and incubation was continued for another $15 \mathrm{~min}$. Then, $5 \mu \mathrm{Ci}$ of $\left[\alpha{ }^{-32}\right.$ P]UTP was added and abortive initiation was allowed to proceed for $15 \mathrm{~min}$ at $30^{\circ} \mathrm{C}$. Transcription reactions were stopped by addition of SDS to $0.2 \%$ and ACU trimer transcripts were extracted with phenol $/ \mathrm{CHCl}_{3}$. To obtain a better resolution between the labeled mononucleotides and the trimers, re- action products were treated with 1.5 units of alkaline phosphatase for $15 \mathrm{~min}$ followed by purification with phenol/ $\mathrm{CHCl}_{3}$ and were analyzed on a $25 \%$ acrylamide sequencing gel.

\section{Electrophoretic mobility shift assay}

Mobility-shift assays were performed essentially as described previously (Kuhn et al. 1993). Reactions $(25 \mu$ l) contained $40 \mathrm{~mm}$ HEPES (pH 8.4), $10 \mathrm{~mm}$ Tris- $\mathrm{HCl}$ (pH 7.9), $4.5 \mathrm{~mm} \mathrm{MgCl}, 80 \mathrm{~mm}$ $\mathrm{KCl}, 0.1 \mathrm{~mm}$ EDTA, $10 \%$ glycerol, $2 \mathrm{~mm}$ dithioerythritol, $2.5 \mu \mathrm{g}$ of BSA, and 0.1 pmoles of a double-strand oligonucleotide lupper strand sequence 5'-GATCTTTTCTATCTGTTCCTATTGGACCTGGAGATAGGTACTG-3') containing a region of ribosomal gene promoter extending from nucleotides -39 to +4 . Protein-DNA complexes were separated by electrophoresis on a native polyacrylamide gel containing $5 \%$ glycerol and running buffer (25 mM Tris- $\mathrm{HCl}(\mathrm{pH} 7.9), 190 \mathrm{~mm}$ glycine, $1 \mathrm{~mm}$ EDTA, $4 \mathrm{mM} \mathrm{MgCl}_{2}$ ]. The anti-Ku antiserum and control serum are as described previously (Kuhn et al. 1993).

\section{Acknowledgments}

We thank J. Craft and J. Steitz for the anti-Ku antiserum. We also thank members of the Jackson and Grummt laboratories for their support and encouragement and Ruth Dendy for help with preparing the manuscript. T.M.G. is supported by a studentship from the Cancer Research Campaign (UK). This work was supported by the Deutsche Forschungsgemeinschaft (Leibniz-Programm and SFB 229|, the Fonds der Chemischen Industrie and by grant SP2143/0101 from the Cancer Research Campaign (UK).

The publication costs of this article were defrayed in part by payment of page charges. This article must therefore be hereby marked "advertisement" in accordance with 18 USC section 1734 solely to indicate this fact.

\section{References}

Anderson, C.W. and S.P. Lees-Miller. 1992. The nuclear serine/ threonine protein kinase DNA-PK. Crit. Rev. Eukaryotic Gene Express. 2: 283-314.

Arias, J.A., S.R. Peterson, and W.S. Dynan. 1991. Promoter-dependent phosphorylation of RNA polymerase II by a template-bound kinase. I. Biol. Chem. 266: 8055-8061.

Bannister, A.J., T.M. Gottlieb, T. Kouzarides, and S.P. Jackson 1993. c-Jun is phosphorylated by the DNA-dependent protein kinase in vitro: Definition of the minimal kinase recognition motif. Nucleic Acids Res. 21: 1289-1295.

Blier, P.R., A.J. Griffith, J. Craft, and J.A. Hardin. 1993. Binding of Ku protein to DNA. J. Biol. Chem. 268: 7594-7601.

Carter, T., I. Vancurova, I. Sun, W. Lou, and S. DeLeon. 1990. A DNA-activated protein kinase from HeLa cell nuclei. Mol. Cell. Biol. 10: 6460-6471.

Comai, L., N. Tanese, and R. Tjian. 1992. The TATA-binding protein and associated factors are integral components of the RNA polymerase I transcription factor, SL1. Cell 68: 965976.

de Vries, E., W. van Driel, W.G. Bergsma, A.C. Arnberg, and P.C. van der Vliet. 1989. HeLa nuclear protein recognizing DNA termini and translocating on DNA forming a regular DNAmultimeric protein complex. J. Mol. Biol. 208: 65-78.

Dvir, A., L.Y. Stein, B.L. Calore, and W.S. Dynan. 1993. Purification and characterisation of a template-associated protein kinase that phosphorylates RNA polymerase II. I. Biol. Chem. 268: 10440-10447. 
Eberhard, D., L. Tora, J.-M. Egly, and I. Grummt. 1993. A TBP. containing multiprotein complex (TIF-IB) mediates transcription specificity of murine RNA polymerase I. Nucleic Acids Res. 21: 4180-4186.

Finnie, N.J., T.M. Gottlieb, T. Blunt, P. Jeggo, and S.P. Jackson. 1995. DNA-PK activity is absent in xrs-6 cells: Implications for site-specific recombination and DNA double-strand break repair. Proc. Natl. Acad. Sci. 92: 320-324.

Francoeur, A.-M., C.L. Peebles, P.T. Gompper, and E.M. Tan, 1986. Identification of $\mathrm{Ki}(\mathrm{Ku}, \mathrm{p} 70 / \mathrm{p} 80)$ autoantigens and analysis of anti-Ki autoantibody reactivity. I. Immunol. 136: $1648-1653$.

Getts, R.C. and T.D. Stamato. 1994. Absence of a Ku-like DNA end-binding activity in the xrs double-strand DNA repair deficient mutant. I. Biol. Chem. 269: 15981-15984.

Gottlieb, T.M. and S.P. Jackson. 1993. The DNA-dependent protein kinase: Requirement for DNA ends and association with $\mathrm{Ku}$ antigen. Cell 72: 131-142.

- 1994. Protein kinases and DNA damage. Trends Biochem. Sci. 19: 500-503.

Hawley, D.K. and R.G. Roeder. 1985. Separation and partial characterization of three functional steps in transcription by human RNA polymerase II. . Biol. Chem. 260: 8163-8172.

Higashiura, M., Y. Shimizu, M. Tanimoto, T. Morita, and T. Yagura. 1992. Immunolocalization of Ku-proteins (p80/p 70): Localization of $\mathrm{p} 70$ to nucleoli and periphery of both interphase nuclei and metaphase chromosomes. Exp. Cell. Res. 201: 444-451.

Jackson, S.P. and R. Tiian. 1989. Purification and analysis of RNA polymerase II transcription factors by using wheat germ agglutinin affinity chromatography. Proc. Natl. Acad. Sci. 86: 1781-1785.

Jackson, S.P., J.J. MacDonald, S. Lees-Miller, and R. Tjian. 1990. GC box binding induces phosphorylation of Spl by a DNAdependent protein kinase. Cell 63: 155-165.

Kuhn, A. and I. Grummt. 1992. Dual role of the nucleolar transcription factor UBF: Trans-activator and antirepressor. Proc. Natl. Acad. Sci. 89: 7340-7344.

Kuhn, A., I. Bartsch, and I. Grummt. 1990. Specific interaction of the murine transcription termination factor TTF I with class-I RNA polymerases. Nature 344: 559-562.

Kuhn, A., V. Stefanovsky, and I. Grummt. 1993. The nucleolar transcription activator $\mathrm{UBF}$ relieves $\mathrm{Ku}$ antigen-mediated repression of mouse ribosomal gene transcription. Nucleic Acids Res. 21: 2057-2063.

Labhart, P. 1994. Identification of two steps during Xenopus ribosomal gene transcription that are sensitive to protein phosphorylation. Mol. Cell. Biol. 14: 2011-2020.

Lees-Miller, S.P., Y-R. Chen, and C.W. Anderson. 1990. Human cells contain a DNA-activated protein kinase that phosphorylates simian virus $40 \mathrm{~T}$ antigen, mouse $\mathrm{p} 53$ and the human $\mathrm{Ku}$ autoantigen. Mol. Cell. Biol. 10: 6472-6481.

McClure, W.R. 1980. Rate-limiting steps in RNA chain initiation. Proc. Natl. Acad. Sci. 77: 5634-5638.

Mimori, T. and J. A. Hardin. 1986. Mechanism of interaction between Ku protein and DNA. I. Biol. Chem. 261: 1037510379.

Morozov, V.E., M. Falzon, C.W. Anderson and E.L. Kuff. 1994. DNA-dependent protein kinase is activated by nicks and larger single-stranded gaps. J. Biol. Chem. 269: 1668416688.

Paillard, S. and F. Strauss. 1991. Analysis of the mechanism of interaction of simian Ku protein with DNA. Nucleic Acids. Res. 19: 5619-5624.

Peterson, S.R., A. Dvir, C.W. Anderson, and W.S. Dynan. 1992. DNA binding provides a signal for phosphorylation of the
RNA polymerase II heptapeptide repeats. Genes \& Dev. 6: $426-438$.

Rathmell, W.K. and G. Chu. 1994. Involvement of the Ku autoantigen in the cellular response to DNA double-strand breaks. Proc. Natl. Acad. Sci, 91: 7623-7627.

Reeves, W.H. and Z.M. Sthoeger. 1989. Molecular cloning of cDNA encoding the $\mathrm{p} 70(\mathrm{Ku})$ lupus autoantigen. $I$. Biol. Chem. 264: 5047-5052.

Rudloff, U., D. Eberhard, L. Tora, H. Stunnenberg, and I. Grummt. 1994. TBP-associated factors interact with DNA and govern species specificity of RNA polymerase I transcription. EMBO I. 13: 2611-2616.

Schnapp, A. and I. Grummt. 1991. Transcription complex formation at the mouse rDNA promoter involves the stepwise association of four transcription factors and RNA polymerase I. J. Biol. Chem. 266: 24588-24595.

Schnapp, A., G. Schnapp, B. Erny, and I. Grummt. 1993. Function of the growth-regulated transcription factor TIF-IA in initiation complex formation at the murine ribosomal gene promoter. Mol. Cell. Biol. 13: 6723-6732.

Schnapp, G., A. Schnapp, H. Rosenbauer, and I. Grummt. 1994 TIF-IC, a factor involved in both transcription initiation and elongation of RNA polymerase I. EMBO I. 13: 4028-4035.

Taccioli, G.E., T.M. Gottlieb, T. Blunt, A. Priestley, J. Demengeot, R. Mizuta, A.R. Lehmann, F.W. Alt, S.P. Jackson, and P.A. Jeggo. 1994. Ku80: Product of the XRCC5 gene and its role in DNA repair and $V(D) J$ recombination. Science 265: 1442-1445.

Yaneva, M. and J. Busch. 1986. A 20 S particle released from deoxyribonuclease-sensitive regions of HeLa cell nuclei contains the 86-kilodalton-70-kilodalton protein complex. Biochemistry 25: 5057-5063.

Yaneva, M. and S. Jhiang. 1991. Expression of the Ku protein during cell proliferation. Biochim. Biophys. Acta 1090: 181187.

Zhang, W.-W. and M. Yaneva. 1992. On the mechanisms of Ku protein binding to DNA. Biochem. Biophys. Res. Commun. 186: $574-579$. 


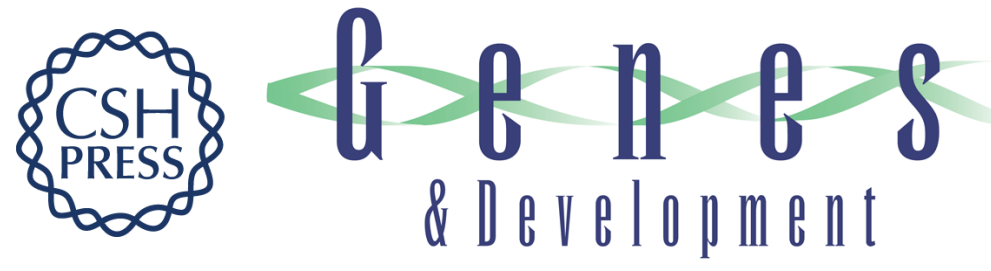

\section{DNA-dependent protein kinase: a potent inhibitor of transcription by RNA polymerase I.}

A Kuhn, T M Gottlieb, S P Jackson, et al.

Genes Dev. 1995, 9:

Access the most recent version at doi:10.1101/gad.9.2.193

References This article cites 38 articles, 21 of which can be accessed free at:

http://genesdev.cshlp.org/content/9/2/193.full.html\#ref-list-1

License

Email Alerting Receive free email alerts when new articles cite this article - sign up in the box at the top Service right corner of the article or click here.

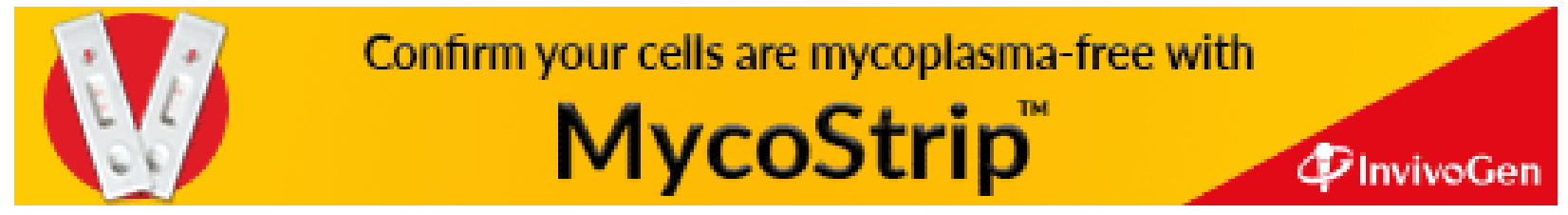

